\title{
Whole-body magnetic resonance imaging: technique, guidelines and key applications
}

\author{
Paul Summers ${ }^{1}$, Giulia Saia ${ }^{1,2}$, Alberto Colombo ${ }^{1}$, Paola Pricolo ${ }^{1}$, Fabio Zugni ${ }^{1}$, Sarah Alessi ${ }^{1}$, Giulia Marvaso ${ }^{3,4}$, \\ Barbara Alicja Jereczek-Fossa ${ }^{3,4}$, Massimo Bellomi ${ }^{1,4}$ and Giuseppe Petralia ${ }^{4,5}$ \\ ${ }^{1}$ Division of Radiology, IEO European Institute of Oncology IRCCS, 20141 Milan, Italy \\ ${ }^{2}$ Advanced Screening Centers, ASC Italia, 24060 Castelli Calepio, Bergamo, Italy \\ ${ }^{3}$ Division of Radiotherapy, IEO European Institute of Oncology IRCCS, 20141 Milan, Italy \\ ${ }^{4}$ Department of Oncology and Hemato-Oncology, University of Milan, 20122 Milan, Italy \\ ${ }^{5}$ Precision Imaging and Research Unit, Department of Medical Imaging and Radiation Sciences, IEO European Institute of Oncology IRCCS, 20141 Milan, Italy
}

\section{Abstract}

Whole-body magnetic resonance imaging (WB-MRI) is an imaging method without ionising radiation that can provide WB coverage with a core protocol of essential imaging contrasts in less than 40 minutes, and it can be complemented with sequences to evaluate specific body regions as needed. In many cases, WB-MRI surpasses bone scintigraphy and computed tomography in detecting and characterising lesions, evaluating their response to therapy and in screening of high-risk patients. Consequently, international guidelines now recommend the use of WB-MRI in the management of patients with multiple myeloma, prostate cancer, melanoma and individuals with certain cancer predisposition syndromes. The use of WB-MRI is also growing for metastatic breast cancer, ovarian cancer and lymphoma as well as for cancer screening amongst the general population. In light of the increasing interest from clinicians and patients in WB-MRI as a radiation-free technique for guiding the management of cancer and for cancer screening, we review its technical basis, current international guidelines for its use and key applications.

Keywords: magnetic resonance imaging, whole-body, diffusion-weighted imaging, oncology

\section{Background}

The introduction of multiple receiver coils and table movement for multi-station scanning in the late 1990s transformed magnetic resonance imaging (MRI) from a segmental to a whole-body (WB) imaging modality [1-3]. Progressive improvements in scanner homogeneity and gradient systems facilitated the introduction of diffusion-weighted imaging (DWI) [4] WB examinations by Takahara et al [5]. Evidence that DWI provides good diagnostic performance in the detection, characterisation and monitoring of therapy of many cancers, consolidated in a first consensus meeting of experts in 2009 [6], has led to DWI having a central role in WB-MRI. Further developments, including accelerated imaging and improved sequence design, have brought improvements in image quality and rendered WB-MRI feasible in a clinically acceptable scan-time of under 40 minutes, opening further opportunities for clinical use [7-9]. In the course of this progression, WB-MRI has become an established part of the management of several cancer histotypes [10-15]. In light of the
Correspondence to: Paul Summers Email: paul.summers@ieo.it

ecancer 2021, 15:1164

https://doi.org/10.3332/ecancer.2021.1164

Published: 07/01/2021

Received: 04/05/2020

Copyright: $@$ the authors; licensee ecancermedicalscience. This is an Open Access article distributed under the terms of the Creative Commons Attribution License (http:// creativecommons.org/licenses/by/3.0), which permits unrestricted use, distribution, and reproduction in any medium, provided the original work is properly cited. 
increasing interest from clinicians and patients in WB-MRI as a radiation-free technique for guiding the management of cancer and for cancer screening, we review its technical basis, current international guidelines for its use and key applications.

\section{Technical considerations}

\section{Sequences and image acquisition}

WB-MRI imaging protocols generally include morphological T1- and T2-weighted sequences along with DWI (Table 1). T1-weighted images are usually obtained with gradient-echo (GRE) Dixon acquisitions [16], producing in- and opposed-phase images that allow the calculation of fat-only and water-only images. These are useful in the detection, characterisation and response assessment of bone metastases. As an alternative, a T1-weighted 3D turbo spin echo (TSE) sequence could be performed [17], but this typically results in a longer acquisition and foregoes the discrimination of water and fat components.

T2-weighted images, acquired using single-shot or half-acquisition turbo spin echo (HASTE) sequences without fat suppression, can be used for the evaluation of disease in organs other than bone [18] with a good trade-off between duration and signal to noise ratio (SNR) [19], and can be helpful in confirming the presence of spinal cord compression [20].

Sagittal T1-weighted TSE and fat-saturated (short tau inversion recovery-STIR) T2-weighted TSE images of the whole spine are used for the detection of vertebral metastases, fractures and spinal cord compression [11, 13].

The final essential component of a WB-MRI protocol is a single-shot diffusion-weighted echo-planar imaging sequence. DWI has become central to WB-MRI due to its ability to detect malignant lesions characterised by high cellularity. Acquiring multiple averages of the DWI data during free-breathing is recommended in order to reduce motion artefacts and increase SNR [21]. At least two $b$-values are needed in order to calculate the corresponding apparent diffusion coefficient (ADC) map for image interpretation and disease response assessment [22]. The lowest $b$-value should be at least $50 \mathrm{~s} / \mathrm{mm}^{2}$ in order to reduce perfusion-related signals, while a high $b$-value in the range between 800 and $1,000 \mathrm{~s} / \mathrm{mm}^{2}$ is recommended $[13,21,22]$. The use of a single diffusion encoding direction with simultaneous application of gradients from all three axes can provide higher SNR by reducing echo times. It may also reduce the blurring that can arise when averaging across images acquired with multiple diffusion encoded directions that are prone to different eddy current induced distortions [23]. The values of ADC in tissues that have anisotropic diffusion (kidneys, and white matter) may, however, be biased with such diffusion encoding, though this is usually of secondary importance for WB-MRI examinations. WB DWI requires a relatively long (10-15 minutes) acquisition time [24, 25]. Obtaining fewer averages of the low $b$-value images, that have intrinsically higher SNR, is one measure for minimising acquisition times.

Coverage of the lung and brain can be incorporated with less than 2 minutes of scanning by using a single breath-hold, short echo-time GRE sequence for evaluation of lung parenchyma [26], and a fluid-attenuated inversion recovery TSE sequence for detection of focal brain lesions or brain oedema. While contrast media is not routinely used in WB-MRI studies, it may be useful for detecting brain metastases or meningeal disease [27]. Depending on clinical context, sequences to evaluate specific body regions can be added to a basic WB-MRI protocol [13]. For example, a post-contrast T1-weighted brain scan is recommended in Li-Fraumeni syndrome (LFS) [28]. For men, axial T2-weighted and DWI sequences targeted to the prostate can be added to provide an all-in-one prostate cancer (PC) staging examination [17, 29].

Following well-established practices for WB positron emission tomography (PET) and computed tomography (CT), WB-MRI acquisitions span from the vertex to mid-thigh. A distinction is that with the patient lying supine, the arms remain adducted. The acquisition should be extended to the feet when performed for diseases that frequently involve the extremities, such as neurofibromatosis [30], or as part of surveillance in cancer predisposition syndromes that favour soft-tissue tumours, such as LFS [31]. The acquisition of WB-MRI in patients affected by multiple myeloma should, instead, span from vertex to knees [11].

Both coronal and axial acquisitions have been used in WB-MRI $[11,17,18]$. The coronal orientation may permit the acquisition of fewer stations and thus reduce scan time. Coronal DWI scans are more likely, however, to suffer from distortion than axial DWI with the same field of view (FOV) and number of slices [32]. Axial acquisition has a further advantage in providing images that can be directly correlated with the conventional cross-sectional anatomy of other modalities. Slice thickness (SLT) should remain consistent across the different sequences to allow efficient image comparison and improve the readability of the examination. The choice of SLT remains somewhat variable; recommendations are for the use of contiguous slices with a thickness between 5 and $7 \mathrm{~mm}[11,13]$. 
Table 1. Sequence components for WB-MRI examinations.

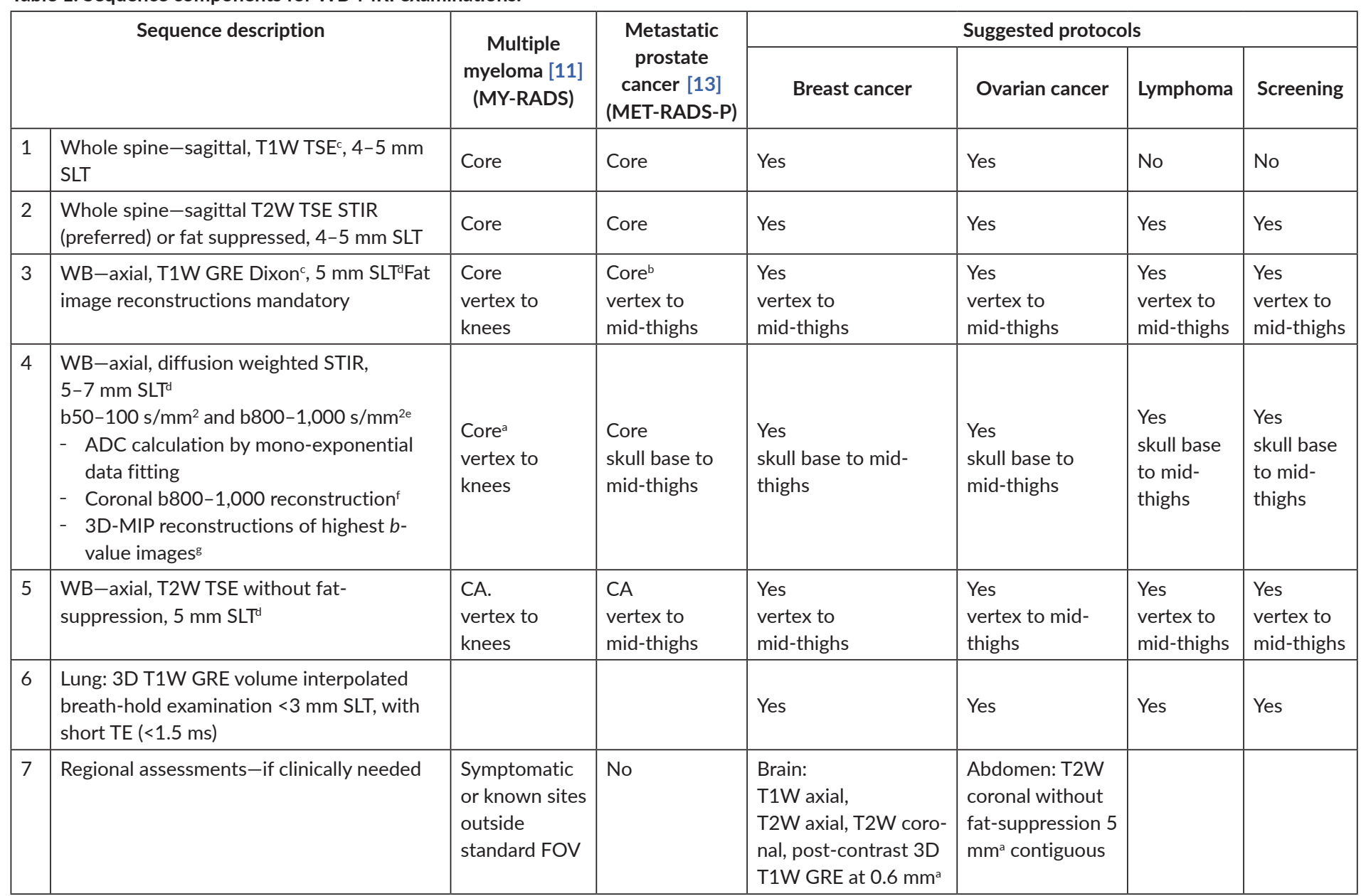

ADC, Apparent diffusion coefficient; CA, Comprehensive assessment; FOV, Field of view; GRE, Gradient echo; MIP, Maximum intensity projection; SLT, Slice thickness; STIR, Short tau inversion recovery; TE, Echo time; TSE, Turbo spin echo; W, Weighted; 3D, Three-dimensional; BC, Breast Cancer; METastasis Reporting and Data System for Prostate Cancer, MET-RADS-P; Myeloma Response Assessment and Diagnosis System, MY-RADS; PC, Prostate Cancer aCoronal acquisition accepted under MY-RADS

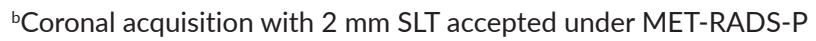

'Alternatively, a 3D T1W TSE allowing multiplanar reformatting may be performed

dSuggest axial with common SLT across WB images to facilitate image review

eAdd b500-600 s/mm² for CA

fb800-1,000 s/mm² DWI images from all stations grouped and reconstructed as $5 \mathrm{~mm}$ contiguous, two-dimensional coronal slices

83D MIP images, displayed rotating around cranio-caudal axis, using an inverted greyscale

WB-MRI can be performed on both 1.5 T and $3 \mathrm{~T}$ scanners, but 1.5 T may be preferred when patients have non-removable metallic prostheses. An advantage of $3 \mathrm{~T}$ scanners is seen in higher SNR, but the lower homogeneity of the static field can reduce the effectiveness of fat saturation leading to a variable SNR in DWI, and increases the risk of 'phase-wrapping' in DIXON scans [33].

The impact of magnetic field inhomogeneity is particularly evident where the superior and inferior limits of successive stations are seen adjacent to each other as happens when viewing the images in anatomic order or in maximum intensity projection (MIP). Attempting to deal with this problem by not shimming the different stations, by applying the same centre frequency to all stations or by limiting the superior-inferior coverage of each station [33] have largely been inadequate. Slice specific shimming [34], on the other hand, has provided consistent reduction, and is to be recommended where available. 
Achieving WB coverage while optimising SNR requires the use of a large number of receiver coils. Given that most modern systems provide a fixed head-coil position, the patient is generally positioned headfirst in the head and neck array coil. In addition, a spine array coil and anterior body coil array(s) are necessary to cover the chest, abdomen and pelvis. If the examination is to be extended to the feet, a lower limb coil should be added in order to maintain a high SNR on the entire body [35]. To avoid time being lost on changeovers of the coils, all coils should be positioned and connected for the duration of the procedure. Because this effectively sandwiches the patient between arrays of coils, it is important to ensure they are comfortable and fully prepared to remain still for the duration of the examination. Preparation should include provision of accurate information about the examination, having the patient change into a single-use gown to avoid hidden metal objects and encouraging them to void their bladder prior to entering the scanner.

Image quality of the Dixon T1- and HASTE T2-weighted images may be improved if the chest and abdomen stations are obtained in breath-hold. The patient should be informed before the examination of the instructions and breath-hold duration. During the examination, they must be able to hear the instructions clearly and communications should be maintained between scans to ensure they are awake when the instructions are given.

\section{Post-processing and quantitative analysis}

Where possible, 'set-up and go' acquisition and post-processing should be used to simplify and accelerate the operational workflow for WB$\mathrm{MRI}$ and reduce the scope for operator errors. To facilitate image reading and reporting, we can unify (compose) the different stations of each sequence into single-stack of images with WB coverage. A series of MIPs of the high $b$-value DW images, rotating around the cranio-caudal axis, allow an 'at-a-glance' overview for disease assessment and lesion detection. Typically, these are displayed in inverse grey scale, thus providing an appearance similar to that of PET.

Relative fat fraction (rF\%) maps have emerged as an objective image-based biomarker of disease [36]. Obtained from T1 GRE Dixon images, $\mathrm{rF} \%$ can provide a quantitative assessment of the distribution of fat within the body, and of oncological relevance, describe bone marrow substitution [37]. The rF\% maps are obtained by dividing the signal intensity of the fat-only images (F) by the sum of the fat-only and wateronly (W) images:

$$
r F \%=\frac{100 \times F}{F+W}
$$

$A D C$ maps are useful for classifying findings and response to therapy [11, 13]. ADC is usually calculated with a monoexponential fitting to the signal intensities in the different $b$-value DWI images:

$$
A D C=-\ln \frac{\left(\frac{S_{\text {high }}}{S_{\text {low }}}\right)}{\left(b_{\text {high }}-b_{\text {low }}\right)},
$$

where $S_{\text {high }}$ and $S_{\text {low }}$ are the signal intensities in the high and low $b$-value images $\left(b_{\text {high }}\right.$ and $\left.b_{\text {low }}\right)$, respectively [38].

The large number of images produced in each WB-MRI examination complicates extracting the quantitative information provided via $\mathrm{rF} \%$ and ADC maps. This necessitates considerable time for reading and reporting, and can increase the risk of misinterpretations. Several semiautomatic segmentation techniques have been developed to assist in distinguishing malignant lesions from healthy tissue and benign findings [37, 39]. A recently proposed tool for WB DWI segmentation combines application of thresholds and manual editing to provide a relatively efficient segmentation of a distributed lesion load (Figure 1). In evaluation of bone marrow and of bone metastases [22, 40] it has shown very good repeatability $[41,42]$ suggesting utility in clinical practice.

Despite considerable progress, WB-MRI still faces a number of technical and practical challenges. Several of these are tied to the quality of the static and radiofrequency magnetic field homogeneities that are available [33]. When attempting to obtain large anatomical coverage in a single scanning station, the static field inhomogeneity can yield distortions that can disrupt anatomy, for example, creating a 'broken spine' artefact between stations on DWI scans, and producing water-fat swaps in the Dixon technique. Radio-frequency inhomogeneities are associated with the use of large numbers of small coils as well as being a function of patient size and composition that can lead to inhomogeneous signal intensity in deep structures or between imaging stations. Both these issues become more pronounced on moving from 1.5T to 3.0T, which is otherwise favourable to reducing the number of averages necessary (and thus scan time) or pursuing higher spatial resolution. With advanced shimming techniques [43], these can be addressed to some extent, though the shimming may add notably to the examination dura- 
tion. Practical issues include a greater sense of being in a close space because of the coverage of the patient with coils, and the need to cope with a long, noisy examination. Nonetheless, when informed well, patients appear to appreciate WB-MRI as an effective imaging technique and see it favourably in comparison to other imaging procedures [44].

\section{Clinical Applications}

WB-MRI has entered a clinical domain dominated by bone scan (BS), (CT), PET and the combination-PET/CT. In WB-MRI, morphological images are produced with a choice of contrasts being available, including DWI associated with changes in the cell density, and T1- and T2-weightings that largely reflect fat and water and fat content, while PET contrast derives from metabolic activity of the administered radioactive tracer. Consequently, differences in sensitivity between WB-MRI and PET are typically attributed to mismatches between hypercellularity or chemical content, and altered metabolism in the chemical pathway targeted by the specific PET tracer. WB-MRI examination times tend to be longer than those of PET, but the examination does not necessitate injection of a radioactive tracer, nor the wait for the tracer to achieve adequate biodistribution for imaging.

\section{Multiple myeloma}

Britain's National Institute for Health and Care Excellence guidance recommends WB-MRI as first-line imaging for suspected myeloma and include it along with fluorodeoxyglucose (FDG)-PET/CT and spine MRI for monitoring in case of serological relapse or disease progression [45]. The Oxford Centre of Evidence-based Medicine similarly recommends WB-MRI for staging all forms of multiple myeloma [10] while the British Society for Haematology recommends WB-MRI for therapy monitoring in multiple myeloma patients [46]. Reflecting the application to therapy monitoring, the Myeloma Response Assessment and Diagnosis System (MY-RADS) was published in 2019 to promote standardisation and reduce variation in acquisition, interpretation and reporting of WB-MRI. MY-RADS describes a core clinical imaging protocol with extension for advanced assessment, a structured reporting template and guidance on defining response assessment categories that incorporates ADC measurements [11].

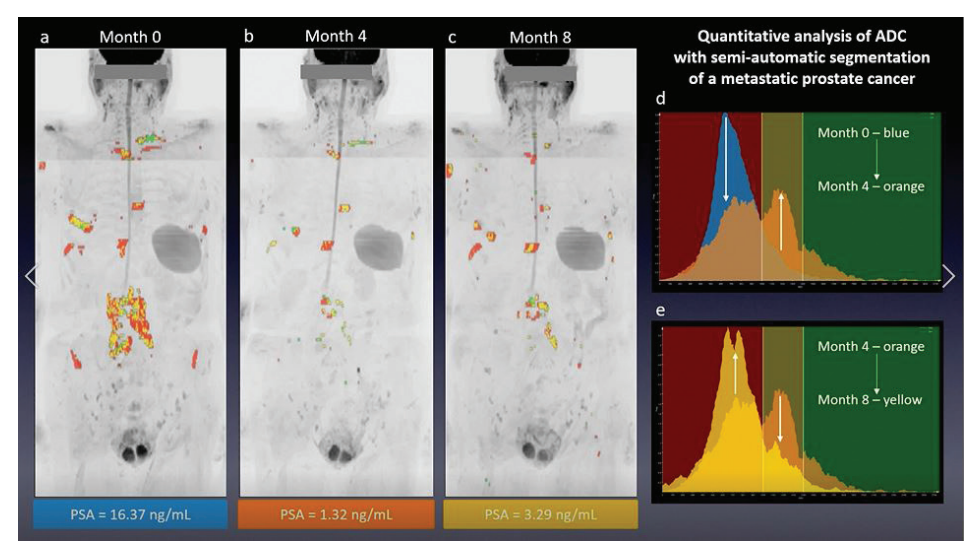

Figure/Video 1. Illustration of the quantitative analysis of ADC via semi-automated segmentation in a patient with metastatic PC. To view this video, click here https://ecancer.org/journal/14/1064-whole-body-magnetic-resonance-imaging-technique-guidelines-and-key-applications. Male with hormonesensitive metastatic PC seen at (a): baseline, (b): 4 month follow-up and (c): 8 month follow-up examinations during hormonal therapy. Overlaid on inverted grey-scale MIPs from b-900 diffusion weighted images are colours representing three ranges of ADC values. Based on MET-RADS-P guidelines [13], the user-defined thresholds ( 1,000 and $\left.1,400 \mu \mathrm{m}^{2} / \mathrm{s}\right)$ define ranges corresponding to: active disease (red) $<1,000 \mu \mathrm{m}^{2} / \mathrm{s}<\mathrm{normal}$ bone/responding lesion (yellow) $<1,400 \mu \mathrm{m}^{2} / \mathrm{s}<$ treated disease/necrotic tissue (green) measured in the bone metastases. These ranges also serve to define the coloured backdrop to comparisons of ADC histograms between successive examinations in (d) and (e). (d): The peak of the (blue) histogram at baseline falls in the active disease range (red band), while the (orange) histogram at first follow-up shows two peaks. The right-most peak, falling in the likely-response range (yellow band) indicating according to the MET-RADS-P guidelines [13], together with a reduction in PSA, this could suggest a positive therapeutic response, but the left-hand peak indicates a residual component of disease is still active. At the second follow-up (yellow histogram), (e): the distribution has returned to the active disease range (red band) and the PSA having increased, is compatible with a predominance of active metastatic disease. 


\section{Prostate cancer}

At initial staging for unfavourable intermediate risk (Gleason Score 4+3) and high-risk PC patients, European Association of Urology (EAU) guidelines recommend at least cross-sectional abdominopelvic imaging and a BS [12]. Similarly, EAU and National Comprehensive Cancer Network (NCCN) guidelines recommend systemic staging with CT and BS for patients with advanced PC (APC). WB-MRI and PET have entered the recent American Society of Clinical Oncology (ASCO) guidelines, where they are indicated for screening where conventional imaging is negative or equivocal [47]. Multiple studies have recognised that MRI is more sensitive than CT and BS for the detection of bone metastases [48, 49]. For this reason, an 'all-in-one' approach (Table 2) that combines multiparametric MRI of the prostate with a WB-MRI study is attractive for local and systemic staging $[13,50]$.

For APC, the ASCO guidelines consider WB-MRI, PET/CT and PET/MRI as alternatives [47]. As prostate membrane specific antigen (PSMA) PET/CT may not inform on tumour viability during androgen receptor inhibition [51], the option of pairing it with FDG-PET has been suggested [52], but bears a significant radiation exposure compared to WB-MRI. Although some studies have shown very good performance of Choline PET for both local and distant disease in staging (e.g. [53]), a 2018 review of the literature suggests it does not perform so well in all circumstances [54]. The Advanced Prostate Cancer Consensus Conference recognises the limitations of BS and CT for bone assessment, and highlights that the flare phenomenon can interfere with the therapy response assessment $[55,56]$. Notably, WB-MRI is not affected by this phenomenon and has clear value in the detection of skeletal related events such as spinal cord compression and fractures. Moreover, for up to $30 \%$ of patients with metastatic castration resistant PC, there is a radiographic progression without clinical/prostate-specific antigen (PSA) progression [57]. Instead, WB-MRI is increasingly used for APC therapy monitoring (Figure 2) and for patients experiencing biochemical recurrence that, in case of oligometastatic disease (one to five oligometastatic lesions), are potentially candidates for local therapy [58]. Evidence from a randomised trial shows that the stereotactic ablative radiotherapy is associated with an improvement in overall survival in patients with oligometastatic PC [59].

Lymph nodes are another common site of PC metastasis and are thus a concern both in staging and in oligoprogression. In a 2016 study by Larbi et al [60], only one-third of enlarged lymph nodes seen on WB-MRI in metastatic prostate cancer patients were within the the regions recommended for external beam radiation therapy or extended lymph node resection, illustrating the importance of whole-body lymph node assessment. MRI and CT, dependent on morphological assessment of nodal disease, have similar but unfortunately limited levels of performance in detection of metastatic nodal detection [61]. Several PET tracers, including 2-deoxy-2-(18F)fluoro-D-glucose, sodium (18F)fluoride, $\left({ }^{11} \mathrm{C}\right)$ choline and $\left({ }^{18} \mathrm{~F}\right)$ choline have been found to perform comparably or worse than MRI and CT for nodal disease, but recent preliminary reports suggest that PET/CT with some (PSMA)-based tracers may permit greater sensitivity without sacrificing specificity [62, 63]. Nonetheless in light of the above-mentioned performance for bone metastases, WB-MRI remains attractive alone or in combination with PSMA PET as the clinical significance of the sub-centimetre nodes that are poorly seen with WB-MRI remains to be established. Moreover, WB-MRI in APC is able to better reflect the heterogeneity of disease [13].

Table 2. 'All-in-one' protocol with WB-MRI for PC staging.

\begin{tabular}{|l|l|l|l|}
\hline \multicolumn{1}{|c|}{ Sequence } & \multicolumn{1}{c|}{ Plane and coverage } & \multicolumn{1}{c|}{ Parameters } & \multicolumn{1}{c|}{ Assessment } \\
\hline T1W TSE & Sagittal whole-spine & $4-5 \mathrm{~mm}^{\mathrm{a}}$ & Bone metastases \\
\hline T1W GRE Dixon & Axial WB & $5 \mathrm{~mm}^{\mathrm{a}}$ & Bone and lymph nodes metastases \\
\hline DWI & Axial WB & $\begin{array}{l}5-7 \mathrm{~mm}^{\mathrm{a}} \\
2 \mathrm{~b} \text {-values: } \\
\text { low: } 50-100 \mathrm{~s} / \mathrm{mm}^{2} \\
\text { high: } 800-1,000 \mathrm{~s} / \mathrm{mm}^{2}\end{array}$ & Bone and lymph nodes metastases \\
\hline T2W TSE & Axial and sagittal & $\begin{array}{l}3 \mathrm{~mm}^{\mathrm{a}} \\
\text { small FOV }\end{array}$ & Prostate \\
\hline DWI & Axostate & $\begin{array}{l}3 \mathrm{~mm}^{\mathrm{a}} \\
\text { small FOV }\end{array}$ & Prostate \\
\hline
\end{tabular}

W, Weighted; TSE, Turbo spin echo; GRE, Gradient echo; DWI, Diffusion weighted imaging; FOV, Field of view ${ }^{\mathrm{a}}$ slice thickness 


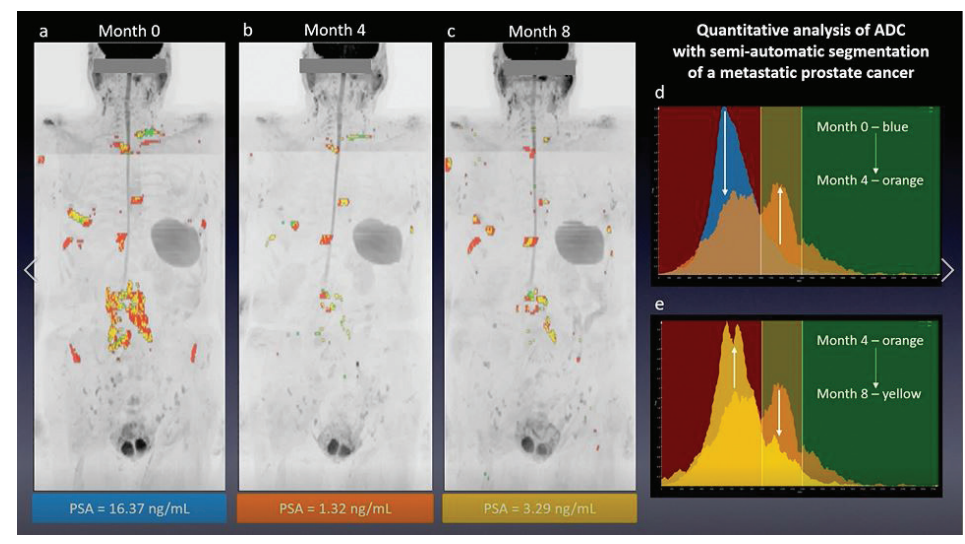

Figure 2. Metastatic PC staging with WB-MRI. A 70-year-old male with high-risk PC (PSA $28 \mathrm{ng} / \mathrm{mL}$, Gleason score (GS) 4+4) seen in (a): coronal inverted grey-scale MIP of b-900 diffusion-weighted images and (b-d): axial b-900 diffusion-weighted images b-900 images from WB-MRI performed for staging. In the 'at-a-glance' disease assessment facilitated by the MIP, WB-MRI shows a clinical pattern of type N1 M1b, involving (b): regional lymph node metastasis (c): a non-regional lymph node metastasis and (d): bone marrow disease.

Standards for image acquisition, interpretation and reporting for clinical care and clinical trials involving APC patients have recently been defined in the Metastasis Reporting and Data System for Prostate Cancer (MET-RADS-P) guidelines [13]. For reporting, these guidelines consider 14 anatomical regions in a baseline template and response assessment criteria for following disease evolution in follow-up.

\section{Melanoma}

The German Dermatological Society and the Dermatologic Cooperative Oncology Group [14, 15] suggest the use of WB-MRI as a valid alternative to contrast-enhanced CT or PET/CT for follow-up of advanced melanoma (stage III or higher) and this is also the recommendation in Swiss guidelines for the treatment and follow-up of cutaneous melanoma [14]. WB-MRI has demonstrated comparable diagnostic performance in the detection of extracranial metastases from melanoma and other tumours in multiple body regions [64, 65]. Lung parenchyma is difficult to study with the conventional T1-, T2- and diffusion-weighted images. Breath-hold, short echo-time, GRE images have been reported to perform well in demonstrating pulmonary lesions greater than $5 \mathrm{~mm}$ [66]. Taking advantage of techniques to improve resolution [64], detection of smaller lesions can be expected; and if negative, low-dose CT of the lungs considered [26]. The study of liver, which has always created concerns for the absence of contrast agent, is performed with DWI and allows detection, even of small metastases, with good sensitivity [67].

\section{Breast cancer}

Currently, international guidelines are mixed as to the which WB imaging techniques to apply and under what conditions (staging, monitoring, recurrence) in the evaluation of breast cancer (BC) patients (see [68] for a summary). CT, BS and FDG-PET dominate European Society for Medical Oncology (ESMO), Royal College of Radiologists (UK) and North American NCCN recommendations for the contexts they cover, with conventional MRI and ultrasound being alternatives for abdominal imaging where appropriate [69-71]. In a direct comparison, the SKELETA Trial [72] showed that WB-MRI provides higher sensitivity for bone metastases arising from breast and PC than BS, single photon emission computed tomography (SPECT) and SPECT-CT (91\% versus $62 \%, 74 \%$ and $85 \%$, respectively), and nearly identical sensitivity to ${ }^{19} \mathrm{~F}-\mathrm{NaF}$ PETCT (93\%) consistent with previous bimodality comparisons [73, 74].

The recent Response Evaluation of Cancer Therapeutics (RESPECT) study [75] monitored treatment response in a cohort of 44 women with metastatic BC using bone scintigraphy, CT and WB-MRI at 12-week intervals. Amongst the 33 patients who arrived at radiological progression during the study, all were seen with WB-MRI at time of first appearance of progression, but only 11 of these were evident on the CT of the corresponding time-point. In the 26 patients whose progression involved bone, just 13 cases were considered in progression on the bone 
scintigraphy at the time of radiological progression. This strongly suggests the suitability of WB-MRI for response assessment in systemic anticancer therapy.

As $B C$ is the second most common cause of brain metastases [76], contrast-enhanced brain MRI should be considered in these patients as the sensitivity has been seen to exceed those of CT and FDG-PET [77]. Liver metastases are also relatively common in BC patients. In the imaging of liver metastases is DWI well-established as outperforming both CT and PET [77, 78].

A subgroup of $B C$ patients for whom WB-MRI is seeing increasing use, is that of young BC patients for whom serial treatment monitoring is necessary. This use of WB-MRI is driven by growing awareness of radiation induced risks, especially for those under 35 years of age [79].

WB-MRI is also establishing an important role in the care of pregnant women with $B C[25,80]$ where systemic staging is mandatory in preparation for childbirth-based therapies. As a technique without ionising radiation and requiring no contrast agent, it is more appropriate than $\mathrm{CT}$ or BS for staging and therapy monitoring in these patients.

\section{Ovarian cancer}

For staging and follow-up of ovarian cancer patients, the European Society of Urogenital Radiology guidelines recommend contrast-enhanced chest-abdomen-pelvis CT [81]. Evidence shows that WB-MRI has higher diagnostic accuracy than CT in determining the malignant nature of ovarian masses [82], as well as for assessing the involvement of mesentery, para-aortic lymph nodes, large bowel and sigmoid-rectum [83]. Furthermore, quantitative analysis using ADC measurements has proven robust to detect early microstructural changes occurring in response to advanced epithelial ovarian cancer [84]. WB-MRI has also been shown to be more accurate than both CT and FDG-PET in the staging of peritoneal lesions ( $91 \%$ versus $75 \%$ and $71 \%$ respectively), and both WB-MRI and FDG-PET to be more accurate than CT in retroperitoneal lymphadenopathy detection (respectively, $87 \%$ and $87 \%$ versus $71 \%$ ) [85]. These promising results suggest that WB-MRI could have a role in the management of ovarian cancer patients.

\section{Lymphoma}

Owing to its high accuracy, FDG-PET/CT is the recommended imaging technique for the staging and follow-up of most common lymphomas (e.g. follicular lymphoma and Hodgkin's lymphoma) [86]. It has been demonstrated, however, that WB-MRI shows higher sensitivity (94\%) for lymphoma detection in patients with variable FDG avidity lymphoma subtypes than FDG-PET/CT (61\%) and contrast-enhanced CT (71\%) [87] (Figure 3). Furthermore, WB-MRI use is emerging in young patients ( $<35$ years) [79] and pregnant women (lymphoma occurring in 1 pregnancy in 1,000$)[88]$ to minimise exposure to ionising radiation.

\section{Lung cancer}

NCCN guidelines recommend the staging of lung cancer with PET/CT and/or total body CT, with the addition of an MRI examination of the brain when needed [89]. The Streamline L study has shown that entirely WB-MRI-based pathways are viable in replacement for standard pathways and require shorter staging time [90]. In lung cancer patients for whom a brain MRI is necessary, integration of this examination with WB-MRI to allow tumor-node-metastasis (TNM) staging in a single sitting is an obvious step for efficiency.

\section{Colorectal cancer}

ESMO and NCCN guidelines both recommend an abdomen-pelvis CT for colon cancer staging, and a chest-abdomen-pelvis CT together with a rectal MRI for rectal cancer staging [91, 92]. The Streamline C study, however, found that WB-MRI-based pathways are a viable replacement for standard pathways in staging patients with colon cancer [93]. Considering this, the role of WB-MRI in the management of both colon and rectal cancer patients can be expected to expand. Moreover, combining WB-MRI systemic staging with rectal MRI for local staging could be of interest as a one-stop-shop for TNM staging of rectal cancer in a single session. 


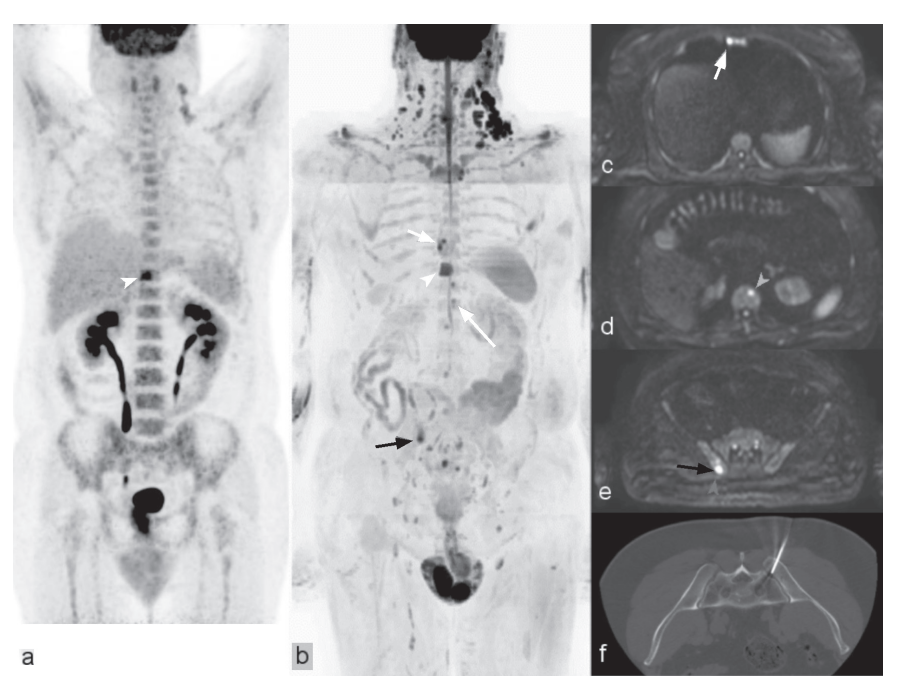

Figure 3. Bone lesions in lymphoma. A 51-year-old male with follicular lymphoma (G1-G2). (a): ${ }^{18} \mathrm{FDG}$-PET for distant staging to have a single bone metastasis of the T10 vertebra (white arrowhead). At WB-MRI 1 month later, oligometastatic disease with multiple bone lesions was evident in (b): the inverted grey scale MIP of the b-900 diffusion weighted images; with corresponding hyperintensities being seen in the axial source images (c-e). In addition to the T10 metastasis (white arrowhead in a and b), a sternal metastasis (smaller white arrow in b and c), a second thoracic vertebral lesion (longer white arrow in $b$ and d) and an iliac lesion (black arrow in b and e). Metastasis in the right iliac bone was confirmed by CT-guided biopsy (f).

\section{Cancer screening}

Due to its spatial resolution and high sensitivity, along with its non-invasive nature and the absence of ionising radiation [94], WB-MRI has emerged as the imaging technique of choice for cancer screening in subjects with cancer predisposition syndromes [28, 31, 95, 96].

According to the American Association for Cancer Research (AACR), as well as a multicentre cohort review involving 13 cohorts and 578 participants, an annual WB-MRI is recommended for patients with LFS as a screening examination [28, 31]. The exam should be complemented with a contrast-enhanced brain MRI and, in women, with a contrast-enhanced breast MRI as well.

For constitutional mismatch repair deficiency (CMMR-D) syndrome, an annual WB-MRI examination from the age of 6 years has been recommended in a consensus statement by the Care for CMMRD Consortium and the International Biallelic Mismatch Repair Deficiency Consortium [95]. For hereditary paraganglioma-pheochromocytoma, on the other hand, the AACR has recommended biennial WB-MRI [96].

The application of WB-MRI to oncological screening in the general population is still a matter of debate. Several reports have demonstrated the use of WB-MRI in asymptomatic individuals [27, 97, 98-106], the largest of which includes 30,000 subjects [107]. Although the methods and results of the studies are too heterogeneous to draw unequivocal conclusions, enough tumours have been diagnosed in asymptomatic subjects to generate clinical interest in the technique and to motivate further research.

\section{Conclusions}

Thanks to a series of improvements in scanner hardware and imaging technique, it is possible to achieve in clinical routine what 20 years ago seemed impossible: a WB-MRI examination that provides a core clinical protocol for WB imaging in little more than half an hour. Continuing developments in imaging strategy and artificial intelligence hold the promise of further improvements and are likely to expand the use cases of WB-MRI in oncology. Existing guidelines demonstrate the central role of WB-MRI examinations with DWI in some oncology applications, but further efforts to standardise acquisition and reporting are warranted to reduce the variation in diagnostic performance. 


\section{Trial registration}

Not applicable.

\section{Conflicts of interest}

Dr Summers is co-owner of QMRI Tech, a company that provides support for medical physics activities and MRI research.

The other authors declare that they have no conflicts of interest.

\section{Funding}

This work was funded by FIEO-CCM, and partially supported by the Italian Ministry of Health with 'Ricerca Corrente' and $5 \times 1000$ funds. The funding bodies did not participate in the planning, design or execution of the study.

\section{References}

1. Carriero A, Scarabino T, and Magarelli N, et al (1997) Whole-body magnetic resonance angiography using a contrast medium. Technical optimization Radiol Med 94 319-324

2. Westenberg JJ, Wasser MN, and van der Geest RJ, et al (1999) Scan optimization of gadolinium contrast-enhanced three-dimensional MRA of peripheral arteries with multiple bolus injections and in vitro validation of stenosis quantification Magn Reson Imaging 17 47-57 https://doi.org/10.1016/S0730-725X(98)00152-0 PMID: 9888398

3. Ruehm SG, Goyen M, and Quick HH, et al (2000) Ganzkorper-MRA auf einer rollenden tischplattform (AngioSURF) RoFo Fortschritte auf dem Gebiet der Rontgenstrahlen und der Bildgeb Verfahren 172 670-674 https://doi.org/10.1055/s-2000-7177

4. Le Bihan D, Breton E, and Lallemand D, et al (1986) MR imaging of intravoxel incoherent motions: application to diffusion and perfusion in neurologic disorders Radiology 161 401-407 https://doi.org/10.1148/radiology.161.2.3763909 PMID: 3763909

5. Takahara T, Imai Y, and Yamashita T, et al (2004) Diffusion weighted whole body imaging with background body signal suppression (DWIBS): technical improvement using free breathing, STIR and high resolution 3D display Radiat Med 22 275-282 PMID: 15468951

6. Padhani AR, Liu G, and Mu-Koh D, et al (2009) Diffusion-weighted magnetic resonance imaging as a cancer biomarker: consensus and recommendations. Neoplasia 11 102-125 https://doi.org/10.1593/neo.81328 PMID: 19186405 PMCID: 2631136

7. Pruessmann KP, Weiger M, and Scheidegger MB, et al (1999) SENSE: sensitivity encoding for fast MRI Magn Reson Med $42952-962$ https://doi.org/10.1002/(SICI)1522-2594(199911)42:5<952::AID-MRM16>3.0.CO;2-S PMID: 10542355

8. Griswold MA, Jakob PM, and Heidemann RM, et al (2002) Generalized autocalibrating partially parallel acquisitions (GRAPPA) Magn Reson Med 47 1202-1210 https://doi.org/10.1002/mrm.10171 PMID: 12111967

9. Katscher U, Börnert P, and Leussler C, et al (2003) Transmit SENSE Magn Reson Med 49 144-150 https://doi.org/10.1002/mrm.10353 PMID: 12509830

10. Oxford Centre for Evidence-based Medicine Levels of evidence [http://www.cebm.net] Date accessed: 1/04/20

11. Messiou C, Hillengass J, and Delorme S, et al (2019) Guidelines for acquisition, interpretation, and reporting of whole-body MRI in myeloma: myeloma response assessment and diagnosis system (MY-RADS) Radiology 291 5-13 https://doi.org/10.1148/ radiol.2019181949 PMID: 30806604 
12. Mottet N, Bellmunt J, and Bolla M, et al (2017) EAU-ESTRO-SIOG guidelines on prostate cancer. part 1: screening, diagnosis, and local treatment with curative intent Eur Urol 71 618-629 https://doi.org/10.1016/j.eururo.2016.08.003

13. Padhani AR, Lecouvet FE, and Tunariu N, et al (2017) METastasis Reporting and data system for prostate cancer: practical guidelines for acquisition, interpretation, and reporting of whole-body magnetic resonance imaging-based evaluations of multiorgan involvement in advanced prostate cancer Eur Urol 71 81-92 https://doi.org/10.1016/j.eururo.2016.05.033 PMCID: 5176005

14. Dummer R, Siano M, and Hunger RE, et al (2016) The updated Swiss guidelines 2016 for the treatment and follow-up of cutaneous melanoma Swiss Med Wkly 2016146 w14279 https://doi.org/10.4414/smw.2016.14279 PMID: 26901103

15. Pflugfelder A, Kochs C, and Blum A, et al (2013) Malignant melanoma s3-guideline "diagnosis, therapy and follow-up of melanoma" JDDG J. der Dtsch Dermatologischen Gesellschaft 11 1-116 https://doi.org/10.1111/ddg.12113_suppl

16. Dixon WT (1984) Simple proton spectroscopic imaging Radiology 153 189-194 https://doi.org/10.1148/radiology.153.1.6089263 PMID: 6089263

17. Schmidt GP, Reiser MF, and Baur-Melnyk A (2009) Whole-body MRI for the staging and follow-up of patients with metastasis Eur J Radiol 70 393-400 https://doi.org/10.1016/j.ejrad.2009.03.045 PMID: 19457631

18. Lee K, Park HY, and Kim KW, et al (2019) Advances in whole body MRI for musculoskeletal imaging: diffusion-weighted imaging J Clin Orthop Trauma 10 680-686 https://doi.org/10.1016/j.jcot.2019.05.018 PMID: 31316239 PMCID: 6611843

19. Fast, Versatile and Cost-Effective FSE MR Imaging: Technical Considerations and Clinical Application (Phoenix: Barrow Neurological Institute) [ttps://www.barrowneuro.org/education/grand-rounds-publications-and-media/barrow-quarterly/volume-16-no-2-2000/fast-versatile-and-cost-effective-fse-mr-imaging-technical-considerations-and-clinical-applications/]

20. Goldberg AL and Kershah SM. (2010) Advances in imaging of vertebral and spinal cord injury J Spinal Cord Med 33 105-116 https://doi. org/10.1080/10790268.2010.11689685 PMID: 20486529 PMCID: 2869279

21. Petralia G, Padhani AR, and Pricolo P, et al (2019) Whole-body magnetic resonance imaging (WB-MRI) in oncology: recommendations and key uses Radiol Medica 124 218-233 https://doi.org/10.1007/s11547-018-0955-7

22. Padhani AR, Makris A, and Gall P, et al (2014) Therapy monitoring of skeletal metastases with whole-body diffusion MRI J Magn Reson Imaging 39 1049-1078 https://doi.org/10.1002/jmri.24548 PMID: 24510426

23. Hectors SJ, Wagner M, and Corcuera-Solano I, et al (2016) Comparison between 3-scan trace and diagonal body diffusion-weighted imaging acquisitions: a phantom and volunteer study Tomography 2 411-420 https://doi.org/10.18383/j.tom.2016.00229

24. Zugni F, Ruju F, and Pricolo P, et al (2018) The added value of whole-body magnetic resonance imaging in the management of patients with advanced breast cancer PLoS One 13 1-16 https://doi.org/10.1371/journal.pone.0205251

25. Peccatori FA, Codacci-Pisanelli G, and Del Grande M, et al (2017) Whole body MRI for systemic staging of breast cancer in pregnant women Breast 35 177-181 https://doi.org/10.1016/j.breast.2017.07.014 PMID: 28756339

26. Petralia G and Padhani AR (2018) Whole-body magnetic resonance imaging in oncology: uses and indications Magn Reson Imaging Clin N Am 26 495-507 https://doi.org/10.1016/j.mric.2018.06.003 PMID: 30316463

27. Cieszanowski A, Maj E, and Kulisiewicz P, et al (2014) Non-contrast-enhanced whole-body magnetic resonance imaging in the general population: the incidence of abnormal findings in patients 50 years old and younger compared to older subjects PLoS One 9 e107840 https://doi.org/10.1371/journal.pone.0107840 PMID: 25259581 PMCID: 4178037

28. Kratz CP, Achatz MI, and Brugières L, et al (2017) Cancer screening recommendations for individuals with Li-Fraumeni syndrome Clin Cancer Res 23 e38-e45 https://doi.org/10.1158/1078-0432.CCR-17-0408 PMID: 28572266

29. Kirkham APS, Haslam P, and Keanie JY, et al (2013) Prostate MRI: who, when, and how? Report from a UK consensus meeting Clin Radiol 68 1016-1023 https://doi.org/10.1016/j.crad.2013.03.030 PMID: 23827086 
30. Cai W, Kassarjian A, and Bredella MA, et al (2009) Tumor burden in patients with neurofibromatosis types 1 and 2 and Schwannomatosis: determination on whole-body MR images Radiology 250 665-673 https://doi.org/10.1148/radiol.2503080700 PMID: 19244040

31. Ballinger ML, Best A, Mai PL, et al (2017) Baseline surveillance in Li-Fraumeni syndrome using whole-body magnetic resonance imaging JAMA Oncol 31634 https://doi.org/10.1001/jamaoncol.2017.1968 PMID: 28772291 PMCID: 5824277

32. Huang $\mathrm{Y}$, Zhang $\mathrm{X}$, and Guo H, et al (2020) Phase-constrained reconstruction of high-resolution multi-shot diffusion weighted image J Magn Reson 312106690 https://doi.org/10.1016/j.jmr.2020.106690 PMID: 32066067

33. Koh D-M, Blackledge M, and Padhani AR, et al (2012) Whole-body diffusion-weighted mri: tips, tricks, and pitfalls Am J Roentgenol 199 252-262 https://doi.org/10.2214/AJR.11.7866

34. Sasaki M, Tozaki M, and Maruyama K, et al (2018) Does integrated shimming improve lesion detection in whole-body diffusion-weighted examinations of patients with breast cancer? Jpn J Radiol 36 736-743 https://doi.org/10.1007/s11604-018-0781-9 PMID: 30264217

35. Schmidt GP, Schoenberg SO, and Reiser MF, et al (2005) Whole-body MR imaging of bone marrow Eur J Radiol 55 33-40 https://doi. org/10.1016/j.ejrad.2005.01.019 PMID: 15950099

36. Bray TJP, Chouhan MD, and Punwani S, et al (2017) Fat fraction mapping using magnetic resonance imaging: insight into pathophysiology Br J Radiol 20170344 https://doi.org/10.1259/bjr.20170344 PMID: 28936896 PMCID: 6223159

37. Lavdas I, Glocker B, and Kamnitsas K, et al (2017) Fully automatic, multiorgan segmentation in normal whole body magnetic resonance imaging (MRI), using classification forests (CFS), convolutional neural networks (CNNs), and a multi-atlas (MA) approach Med Phys 44 5210-5220 https://doi.org/10.1002/mp.12492 PMID: 28756622

38. Park SY, Kim CK, and Park BK, et al (2014) Comparison of apparent diffusion coefficient calculation between two-point and multipoint b value analyses in prostate cancer and benign prostate tissue at $3 \mathrm{~T}$ : preliminary experience Am J Roentgenol $203 \mathrm{~W} 287-\mathrm{W} 294$ https:// doi.org/10.2214/AJR.13.11818

39. Ceranka J, Verga S, and Kvasnytsia M, et al (2020) Multi-atlas segmentation of the skeleton from whole-body MRI-Impact of iterative background masking Magn Reson Med 83 1851-1862 https://doi.org/10.1002/mrm.28042

40. Blackledge MD, Collins DJ, and Tunariu N, et al (2014) Assessment of treatment response by total tumor volume and global apparent diffusion coefficient using diffusion-weighted MRI in patients with metastatic bone disease: a feasibility study PLoS One 9 1-8 https:// doi.org/10.1371/journal.pone.0091779

41. Blackledge MD, Tunariu N, and Orton MR, et al (2016) Inter- and intra-observer repeatability of quantitative whole-body, diffusionweighted imaging (WBDWI) in metastatic bone disease PLoS One 11 e0153840 https://doi.org/10.1371/journal.pone.0153840 PMID: 27123931 PMCID: 4849763

42. Perez-Lopez R, Mateo J, and Mossop H, et al (2017) Diffusion-weighted imaging as a treatment response biomarker for evaluating bone metastases in prostate cancer: a pilot study Radiology 283 168-177 https://doi.org/10.1148/radiol.2016160646

43. Zhang $\mathrm{H}$, Xue $\mathrm{H}$, and Alto $\mathrm{S}$, et al (2016) Integrated shimming improves lesion detection in whole-body diffusion-weighted examinations of patients with plasma disorder at 3 T Invest Radiol 51(5) 297-305 https://doi.org/10.1097/RLI.00000000000000238

44. Oliveri S, Paola P, and Silvia P, et al (2018) Investigating cancer patient acceptance of Whole Body MRI Clin Imaging 52 246-251 https:// doi.org/10.1016/j.clinimag.2018.08.004 PMID: $\underline{30170274}$

45. National Institute for health and Care Excellence (2016) Guideline NG35: myeloma: diagnosis and management [www.nice.org.uk/guidance/ng35] Date accessed: 21/10/20

46. Chantry A, Kazmi M, and Barrington S, et al (2017) Guidelines for the use of imaging in the management of patients with myeloma $\mathrm{Br} J$ Haematol 2017 178(3) 380-393 https://doi.org/10.1111/bjh.14827 PMID: 28677897 
47. Trabulsi EJ, Rumble RB, Jadvar H, et al. (2020) Optimum imaging strategies for advanced prostate cancer: ASCO guideline J Clin Oncol 2020 38(17) 1963-1996 https://doi.org/10.1200/JCO.19.02757 PMID: 31940221

48. Shen G, Deng H, and Hu S, et al (2014) Comparison of choline-PET/CT, MRI, SPECT, and bone scintigraphy in the diagnosis of bone metastases in patients with prostate cancer: a meta-analysis Skeletal Radiol 43 1503-1513 https://doi.org/10.1007/s00256-0141903-9 PMID: 24841276

49. Woo S, Suh CH, and Kim SY, et al (2018) Diagnostic Performance of magnetic resonance imaging for the detection of bone metastasis in prostate cancer: a systematic review and meta-analysis Eur Urol 73 81-91 https://doi.org/10.1016/j.eururo.2017.03.042

50. Johnston EW, Latifoltojar A, and Sidhu HS, et al (2019) Multiparametric whole-body 3.0-T MRI in newly diagnosed intermediate- and high-risk prostate cancer: diagnostic accuracy and interobserver agreement for nodal and metastatic staging Eur Radiol 29 $3159-3169$ https://doi.org/10.1007/s00330-018-5813-4 PMCID: 6510859

51. Meller B, Bremmer F, and Sahlmann CO, et al (2015) Alterations in androgen deprivation enhanced prostate-specific membrane antigen (PSMA) expression in prostate cancer cells as a target for diagnostics and therapy EJNMMI Res 5 66 https://doi.org/10.1186/s13550015-0145-8 PMID: 26576996 PMCID: 4648835

52. Wang B, Liu C, and Wei Y, et al (2020) A prospective trial of 68 Ga-PSMA and 18 F-FDG PET/CT in nonmetastatic prostate cancer patients with an Early PSA progression during castration Clin Cancer Res 26 4551-4558 https://doi.org/10.1158/1078-0432.CCR-200587 PMID: 32527944

53. Metser U, Berlin A, and Halankar J, et al (2018) 18F-fluorocholine PET whole-body MRI in the staging of high-risk prostate cancer Am J Roentgenol 210 635-640 https://doi.org/10.2214/AJR.17.18567

54. Li R, Ravizzini GC, and Gorin MA, et al (2018) The use of PET/CT in prostate cancer. Prostate Cancer Prostatic Dis $214-21$ https://doi. org/10.1038/s41391-017-0007-8

55. Gillessen S, Attard G, and Beer TM, et al (2018) Management of patients with advanced prostate cancer: the report of the advanced prostate cancer consensus conference APCCC 2017 Eur Urol 73 178-211 https://doi.org/10.1016/j.eururo.2017.06.002

56. Gillessen S, Attard G, and Beer TM, et al (2020) Management of patients with advanced prostate cancer: report of the advanced prostate cancer consensus conference 2019 Eur Urol 77(4) 508-547 https://doi.org/10.1016/j.eururo.2020.01.012 PMID: 32001144

57. Morris MJ, Molina A, Small EJ, et al. (2015) Radiographic progression-free survival as a response biomarker in metastatic castration-resistant prostate cancer: COU-AA-302 results J Clin Oncol 33 1356-1363 https://doi.org/10.1200/JCO.2014.55.3875 PMID: 25624432 PMCID: 4881370

58. Marvaso G, Ciardo D, and Corrao G, et al (2019) Radioablation +/- hormonotherapy for prostate cancer oligorecurrences (Radiosa trial): potential of imaging and biology (AIRC IG-22159) BMC Cancer 19903 https://doi.org/10.1186/s12885-019-6117-z

59. Palma DA, Olson R, and Harrow S, et al (2019) Stereotactic ablative radiotherapy versus standard of care palliative treatment in patients with oligometastatic cancers (SABR-COMET): a randomised, phase 2, open-label trial Lancet 393 2051-2058 https://doi.org/10.1016/ S0140-6736(18)32487-5 PMID: 30982687

60. Larbi A, Dallaudière B, Pasoglou V, et al. (2016) Whole body MRI (WB-MRI) assessment of metastatic spread in prostate cancer: therapeutic perspectives on targeted management of oligometastatic disease Prostate 76(11) 1024-1033 https://doi.org/10.1002/ pros.23196 PMID: 27197649

61. Hövels AM, Heesakkers RAM, and Adang EM, et al (2008) The diagnostic accuracy of CT and MRI in the staging of pelvic lymph nodes in patients with prostate cancer: a meta-analysis Clin Radiol 63(4) 387-395 https://doi.org/10.1016/j.crad.2007.05.022 PMID: 18325358

62. Zarzour JG, Galgano S, and McConathy J, et al (2017) Lymph node imaging in initial staging of prostate cancer: an overview and update World J Radiol 9(10) 389-399 https://doi.org/10.4329/wjr.v9.i10.389 PMID: 29104741 PMCID: 5661167 
63. Meißner S, Janssen JC, and Prasad V, et al (2019) Accuracy of standard clinical 3T prostate MRI for pelvic lymph node staging: comparison to 68 Ga-PSMA PET-CT Sci Rep 9 https://doi.org/10.1038/s41598-019-46386-3

64. Petralia G, Padhani A, and Summers P, et al (2013) Whole-body diffusion-weighted imaging: Is it all we need for detecting metastases in melanoma patients? Eur Radiol 23 3466-3476 https://doi.org/10.1007/s00330-013-2968-x PMID: 23884300

65. Ciliberto M, Maggi F, and Treglia G, et al (2013) Comparison between whole-body MRI and Fluorine-18-Fluorodeoxyglucose PET or PET / CT in oncology : a systematic review 47(3) 206-218 https://doi.org/10.2478/raon-2013-0007 PMID: 24133384 PMCID: 3794875

66. Müller-Horvat C, Radny P, and Eigentler TK, et al (2006) Prospective comparison of the impact on treatment decisions of whole-body magnetic resonance imaging and computed tomography in patients with metastatic malignant melanoma Eur J Cancer 42(3) 342-350 https://doi.org/10.1016/j.ejca.2005.10.008

67. Kenis C, Deckers F, and De Foer B, et al (2012) Diagnosis of liver metastases: Can diffusion-weighted imaging (DWI) be used as a stand alone sequence? Eur J Radiol 81 1016-1023 https://doi.org/10.1016/j.ejrad.2011.02.019

68. Pesapane F, Downey K, and Rotili A, et al (2020) Imaging diagnosis of metastatic breast cancer Insights Imaging 1179 https://doi. org/10.1186/s13244-020-00885-4 PMID: 32548731 PMCID: 7297923

69. Cardoso F, Kyriakides S, and Ohno S, et al (2019) Early breast cancer: ESMO Clinical Practice Guidelines for diagnosis, treatment and follow-up Ann Oncol 30(8) 1194-1220 https://doi.org/10.1093/annonc/mdz173 PMID: 31161190

70. Kay C, Roberts A, and Guthrie A, et al (2014) Pancreas Recommendations for Cross-Sectional Imaging in Cancer Management 2nd edn. ed T Nicholson (London: Royal College Radiologists)

71. Bevers TB, Helvie M, and Bonaccio E, et al (2018) Breast cancer screening and diagnosis, version 3.2018, NCCN clinical practice guidelines in oncology J Natl Compr Cancer Netw 16 1362-1389 https://doi.org/10.6004/jnccn.2018.0083

72. Jambor I, Kuisma A, and Ramadan S, et al. (2016) Prospective evaluation of planar bone scintigraphy, SPECT, SPECT/CT, 18F-NaF PET/ CT and whole body 1.5T MRI, including DWI, for the detection of bone metastases in high risk breast and prostate cancer patients: SKELETA clinical trial Acta Oncol (Madr) 55(1) 59-67 https://doi.org/10.3109/0284186X.2015.1027411

73. Yang HL, Liu T, and Wang XM, et al (2011) Diagnosis of bone metastases: A meta-analysis comparing 18FDG PET, CT, MRI and bone scintigraphy Eur Radiol 21 2604-2617 https://doi.org/10.1007/s00330-011-2221-4 PMID: 21887484

74. Gutzeit A, Doert A, and Froehlich JM, et al (2010) Comparison of diffusion-weighted whole body MRI and skeletal scintigraphy for the detection of bone metastases in patients with prostate or breast carcinoma Skeletal Radiol 39 333-343 https://doi.org/10.1007/ s00256-009-0789-4 PMID: 20205350

75. Kosmin M, Padhani MAR, and Gogbashian MA, et al (2020) Comparison of whole-body MRI, CT, and bone scintigraphy for response evaluation of cancer therapeutics in metastatic breast cancer to bone Radiology 297(3) https://doi.org/10.1148/radiol.2020192683 PMID: 33078998

76. Barnholtz-Sloan JS, Sloan AE, and Davis FG, et al (2004) Incidence proportions of brain metastases in patients diagnosed (1973 to 2001) in the Metropolitan Detroit Cancer Surveillance System J Clin Oncol 22(14) 2865-2872 https://doi.org/10.1200/JCO.2004.12.149 PMID: 15254054

77. Schmidt GP, Baur-Melnyk A, Haug A, et al (2008) Comprehensive imaging of tumor recurrence in breast cancer patients using wholebody MRI at 1.5 and 3 T compared to FDG-PET-CT Eur J Radiol 2008 https://doi.org/10.1016/j.ejrad.2007.10.021

78. Chandarana H and Taouli B (2010) Diffusion and perfusion imaging of the liver Eur J Radiol 76 348-358 https://doi.org/10.1016/j. ejrad.2010.03.016 PMID: 20399054 
79. Brenner H, Gondos A, and Pulte D (2009) Survival expectations of patients diagnosed with Hodgkin's Lymphoma in 2006-2010 Oncologist 14 806-813 https://doi.org/10.1634/theoncologist.2008-0285 PMID: 19648314

80. Han SN, Amant F, and Michielsen K, et al (2018) Feasibility of whole-body diffusion-weighted MRI for detection of primary tumour, nodal and distant metastases in women with cancer during pregnancy: a pilot study Eur Radiol 28(5) 1862-1874 https://doi.org/10.1007/ s00330-017-5126-z

81. Forstner R, Sala E, and Kinkel K, et al (2010) ESUR guidelines: ovarian cancer staging and follow-up Eur Radiol $202773-2780$ https:// doi.org/10.1007/s00330-010-1886-4 PMID: 20839002

82. Michielsen K, Dresen R, and Vanslembrouck R, et al (2017) Diagnostic value of whole body diffusion-weighted MRI compared to computed tomography for pre-operative assessment of patients suspected for ovarian cancer Eur J Cancer 83 88-98 https://doi. org/10.1016/j.ejca.2017.06.010 PMID: 28734146

83. Rizzo S, De Piano F, and Buscarino V, et al (2020) Pre-operative evaluation of epithelial ovarian cancer patients: role of whole body diffusion weighted imaging MR and CT scans in the selection of patients suitable for primary debulking surgery. A single-centre study Eur J Radiol 123108786 https://doi.org/10.1016/j.ejrad.2019.108786

84. Winfield JM, Wakefield JC, and Dolling D, et al (2019) Diffusion-weighted MRI in advanced epithelial ovarian cancer: apparent diffusion coefficient as a response marker Radiology 293 374-383 https://doi.org/10.1148/radiol.2019190545 PMID: 31573402

85. Michielsen K, Vergote I, and Op De Beeck K, et al (2014) Whole-body MRI with diffusion-weighted sequence for staging of patients with suspected ovarian cancer: a clinical feasibility study in comparison to CT and FDG-PET/CT Eur Radiol 24 889-901 https://doi. org/10.1007/s00330-013-3083-8

86. Barrington SF, Mikhaeel NG, and Kostakoglu L, et al (2014) Role of imaging in the staging and response assessment of lymphoma: consensus of the International Conference on Malignant Lymphomas Imaging Working Group J Clin Oncol 32 3048-3058 https://doi. org/10.1200/JCO.2013.53.5229 PMID: 25113771 PMCID: $\underline{5015423}$

87. Mayerhoefer ME, Karanikas G, and Kletter K, et al (2014) Evaluation of diffusion-weighted MRI for pretherapeutic assessment and staging of lymphoma: results of a prospective study in 140 patients Clin Cancer Res 20(11) 2984-2993 https://doi.org/10.1158/10780432.CCR-13-3355 PMID: 24696320

88. Pohlman B and Macklis RM. (2000) Lymphoma and pregnancy Semin Oncol 27(6) 657-666 PMID: 11130473

89. Ettinger DS, Aisner DL, and Wood DE, et al (2018) NCCN guidelines $®$ insights non-small cell lung cancer, version 5.2018 featured updates to the NCCN guidelines JNCCN J Natl Compr Cancer Netw 16 807-821 https://doi.org/10.6004/jnccn.2018.0062

90. Taylor SA, Mallett S, and Ball S, et al (2019) Diagnostic accuracy of whole-body MRI versus standard imaging pathways for metastatic disease in newly diagnosed non-small-cell lung cancer: the prospective Streamline L trial Lancet Respir Med $7523-532$ https://doi. org/10.1016/S2213-2600(19)30090-6 PMID: 31080129 PMCID: 6529610

91. Glimelius B, Tiret E, and Cervantes A, et al (2013) Rectal cancer: ESMO clinical practice guidelines for diagnosis, treatment and followup Ann Oncol 24 vi81-vi88 https://doi.org/10.1093/annonc/mdt240 PMID: 24078665

92. Benson AB, Venook AP, and Al-Hawary MM, et al (2018) NCCN guidelines $₫$ insights colon cancer, version 2.2018 featured updates to the NCCN guidelines JNCCN J Natl Compr Cancer Netw 16 359-369 https://doi.org/10.6004/jnccn.2018.0021

93. Taylor SA, Mallett S, and Beare S, et al (2019) Diagnostic accuracy of whole-body MRI versus standard imaging pathways for metastatic disease in newly diagnosed colorectal cancer: the prospective Streamline C trial Lancet Gastroenterol Hepatol 4 529-537 https://doi. org/10.1016/S2468-1253(19)30056-1 PMID: 31080095 PMCID: 6547166

94. Li B, Li Q, and Nie W, et al (2014) Diagnostic value of whole-body diffusion-weighted magnetic resonance imaging for detection of primary and metastatic malignancies: a meta-analysis Eur J Radiol 83 338-344 https://doi.org/10.1016/j.ejrad.2013.11.017 
95. Tabori U, Hansford JR, and Achatz MI, et al (2017) Clinical management and tumor surveillance recommendations of inherited mismatch repair deficiency in childhood Clin Cancer Res 23 e32-e37 https://doi.org/10.1158/1078-0432.CCR-17-0574 PMID: 28572265

96. Rednam SP, Erez A, and Druker H, et al (2017) Von Hippel-Lindau and hereditary pheochromocytoma/paraganglioma syndromes: Clinical features, genetics, and surveillance recommendations in childhood Clin Cancer Res 23 e68-e75 https://doi.org/10.1158/10780432.CCR-17-0547 PMID: 28620007

97. Baumgart D and Egelhof T. (2007) Präventives Ganzkörperscreening unter Einbeziehung moderner Bildgebung mit Hilfe der Magnetresonanztomographie. Herz Kardiovaskuläre Erkrankungen 32 387-394 https://doi.org/10.1007/s00059-007-3020-1

98. Goehde SC, Hunold P, and Vogt FM, et al (2005) Full-Body cardiovascular and tumor mri for early detection of disease: feasibility and initial experience in 298 subjects Am J Roentgenol 184 598-611 https://doi.org/10.2214/ajr.184.2.01840598

99. Hegenscheid K, Seipel R, and Schmidt CO, et al (2013) Potentially relevant incidental findings on research whole-body MRI in the general adult population: frequencies and management Eur Radiol 23 816-826 https://doi.org/10.1007/s00330-012-2636-6

100.Lee SY, Park HJ, and Kim MS, et al (2018) An initial experience with the use of whole body MRI for cancer screening and regular health checks PLoS One 13 e0206681 https://doi.org/10.1371/journal.pone.0206681 PMID: 30462666 PMCID: 6248944

101.Lo GG, Ai V, and Au-Yeung KM, et al (2008) Magnetic resonance whole body imaging at 3 Tesla: feasibility and findings in a cohort of asymptomatic medical doctors Hong Kong Med J 14 90-96 PMID: 18382014

102.Perkins BA, Caskey CT, and Brar P, et al (2018) Precision medicine screening using whole-genome sequencing and advanced imaging to identify disease risk in adults Proc Natl Acad Sci 115 3686-3691 https://doi.org/10.1073/pnas.1706096114 PMID: 29555771 PMCID: 5889622

103.Saya S, Killick E, and Thomas S, et al (2017) Baseline results from the UK SIGNIFY study: a whole-body MRI screening study in TP53 mutation carriers and matched controls Fam Cancer 16 433-440 https://doi.org/10.1007/s10689-017-9965-1 PMID: 28091804 PMCID: 5487773

104.Takahara T, Kwee T, and Kibune S, et al (2010) Whole-body MRI using a sliding table and repositioning surface coil approach Eur Radiol 20 1366-1373 https://doi.org/10.1007/s00330-009-1674-1 PMCID: 2861758

105.Tarnoki DL, Tarnoki AD, and Richter A, et al (2015) Clinical value of whole-body magnetic resonance imaging in health screening of general adult population Radiol Oncol 49 10-16 https://doi.org/10.2478/raon-2014-0031 PMID: 25810696 PMCID: 4362601

106. Ulus S, Suleyman E, and Aksoy Ozcan U, et al (2016) Whole-body mri screening in asymptomatic subjects; preliminary experience and long-term follow-up findings Polish J Radiol 81 407-414 https://doi.org/10.12659/PJR.897570

107.Bamberg F, Kauczor H-U, and Weckbach S, et al (2015) Whole-body MR imaging in the German National Cohort: rationale, design, and technical background Radiology 277 206-220 https://doi.org/10.1148/radiol.2015142272 PMID: 25989618 